\title{
Laser Induced Periodic Surface Structures on 100Cr6 Steel for Modification of Friction Demonstrated with Stribeck Test
}

\author{
Stefan Rung ${ }^{1}$, Kevin Bokan ${ }^{1}$, Kevin Rutsch ${ }^{1}$, Simon Schwarz ${ }^{1}$, Cemal Esen² and Ralf Hellmann ${ }^{1}$ \\ ${ }^{1}$ Applied Laser and Photonics Group, University of Applied Sciences Aschaffenburg, Wuerzburger \\ Strasse 45, 63743 Aschaffenburg, Germany \\ E-mail: Stefan.rung@h-ab.de \\ ${ }^{2}$ Applied Laser Technologies, Ruhr-University Bochum, Universitaetsstrasse 150, Bochum 44801,
} Germany

\begin{abstract}
In this contribution, we report on surface functionalization by introducing laser induced periodic surface structures on 100Cr6 bearing steel to modify complex tribological properties. The advanced approach of this study is the measurement of the coefficient of friction by performing a ball-on-disk Stribeck test on laser structured surfaces with polytetrafluoroethylene balls in a lubricant environment. The Stribeck test reveals the modified friction behavior using translation speeds up to $10^{6} \mathrm{~mm} / \mathrm{min}$ and load forces between $100 \mathrm{mN}$ and $5000 \mathrm{mN}$. Our results show increased stiction and coefficient of friction for laser structured surfaces in the regime of boundary lubrication which is attributed to the laser induced surface asperities. Decreased coefficient of friction is observed in the regime of mixed and hydrodynamic lubrication, i.e. for velocities higher than $1000 \mathrm{~mm} / \mathrm{min}$, for surfaces covered by laser induced periodic surface structures with low spatial frequency.
\end{abstract}

DOI: 10.2961/jlmn.2018.03.0004

Keywords: laser-induced periodic surface structures; tribology; Stribeck test

\section{Introduction}

Power generation, production engineering and transportation of goods are, from a very fundamental point of view, based on machines which involve a variety of moving parts and interacting surfaces. Ecological and economical aspects, such as energy efficiency, long-term stability and reliability are effectively determined by the friction and wear between interacting surfaces. A recent study reveals that about 23\% of the world's energy consumption and $8120 \mathrm{Mt} /$ year of $\mathrm{CO}_{2}$ emission are caused by these tribological effects, in turn posing the necessity for wear and friction control [1]. While laser based surface functionalization has already been intensively studied during the last decade, femtosecond laser technology has recently expedited surface structuring on different length scales to advance tribological properties [2-5]. In particular, the formation of Laser Induced Periodic Surface Structures (LIPSS) has been investigated on different materials like metals $[6 ; 7]$, dielectrics $[8 ; 9]$ and semiconductors $[10 ; 11]$. These fs-laser induced surface morphologies can be generated in a single-step process and provide multiple possibilities to modify different surfaces properties. Possible applications involve colorization due to diffraction at the periodic structures $[12 ; 13]$, modification of surface wetting properties $[14 ; 15]$, influence on surface cell growth [16; 17] and friction management [18; 19]. Generally, LIPSS are categorized into 3 groups, namely low spatial frequency LIPSS (LSFL), high spatial frequency LIPSS (HSFL) and cone like protrusions (CLP).

In this study, we use LSFL to alter tribological properties. These structures appear upon laser irradiation with linear polarized light. The origin of LSFL is commonly explained by an interference effect of the incident laser light and a surface electro-magnetic wave generated by a laser induced surface plasmon polarition (SPP). Due to this origin, LSFL occur with a spatial periodicity in the range of the used laser wavelength and an orientation perpendicular to the polarization of the laser light. Several research groups have shown that LSFL properties like spatial period, orientation and homogeneity can be also controlled by the applied laser fluence [20;21], pulse to pulse overlap [22] and initial surface roughness of the solid material [23].

While, in general, friction properties of surfaces depend next to the specific materials considered on several properties of the sliding surface itself, the properties of the counter body, the sliding speed, the environmental conditions and the nature of any lubricant, it is obvious that numerous experimental studies are needed to get fully acquainted with the effects of laser induced periodic surface structures on such tribological properties.

From a very general point of view, lubricated friction as investigated here can be categorized into boundary, mixed and hydrodynamic regimes. At the beginning of the relative motion between sliding partners and at low speeds, friction is governed by their physical contact. This may also occur during heavy load conditions. In this regime, the lubrication solvent thickness is smaller than the surface roughness. For bearing and journal materials, e.g., this is the most undesirable regime as it is characterized by a high coefficient of friction (i.e. energy loss) and increased wear.

With increasing sliding speed a wedge or slice of lubricant is created between the surfaces and boundary lubrication is significantly reduced. As the lubricant film thickness increases and asperity contact is reduced, friction decreases leading to a state denoted as mixed lubricant regime. Mixed refers to a state that can be described by an increasing lubricant film thickness and by remaining asperities between the sliding partners being loaded with lubricant. With continuously increasing sliding speed, this condition is translated to 


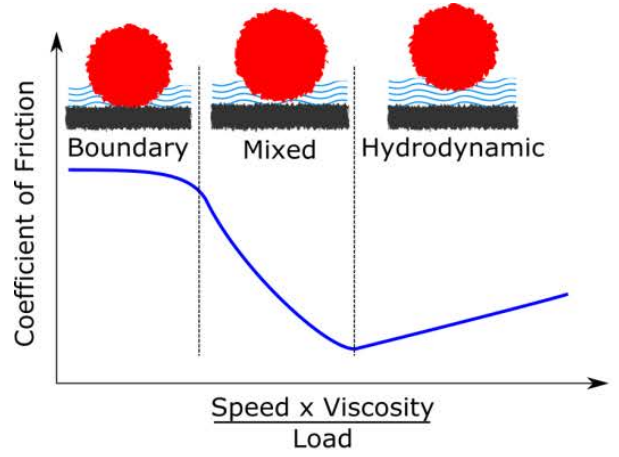

(a)

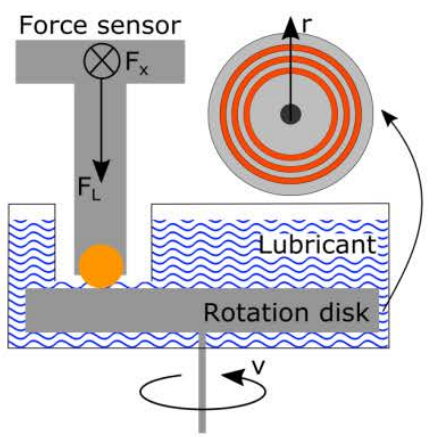

(b)

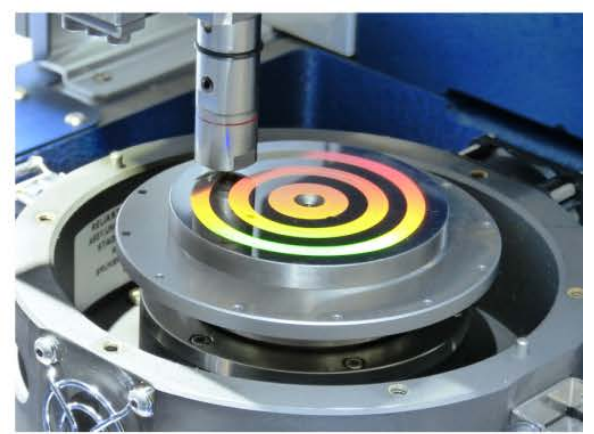

(c)

Fig. 1 (a) Schematic Stribeck curve and (b) the used test setup to measure the coefficient of friction as a function of the sliding speed. (c) shows the implementation of LIPSS covered base plate in the TriboLab (without lubricant camber).

the hydrodynamic or elastohydrodynamic lubrication regime, in which the film thickness increases further towards a full persistent lubricant film clearing the sliding partners. This is the condition to be intended to avoid friction. Friction in this regime is then also governed by the molecular friction within the lubricant itself. With a further increase of the sliding speed, friction can continue to decrease, increase or remain constant depending on lubricant viscosity and temperature. This fundamental behavior was already elaborated in an early work by Stribeck [24].

Bonse et al. [25] reported on the friction behavior of LIPSS covered surfaces in comparison to polished surfaces for different materials and lubricants. In particular, as compared to the polished reference the coefficient of friction (COF) was found to be reduced by more than a factor of two using engine oil as lubricant on titanium sample surfaces covered with LIPSS. For steel no significant difference was observed between polished reference and LIPSS covered surfaces. In addition, no significant influence of the orientation of LIPSS on the friction was found. Eichstädt et al. [26] report an increased COF on silicon structured by LIPSS, whereas Bonse et al. [27] reported a possible COF reduction of up to $24 \%$ in a ball on disc configuration using $100 \mathrm{Cr} 6$ steel disc and 100Cr6 steel ball, lubricated with engine oil.

The interplay between sliding direction of the counter body and the structure orientation was investigated by Wang et al. [28], reporting a reduction of the COF for sliding perpendicular to the periodic as compared to parallel movement.

In this contribution, we report on the friction behavior of LSFL covered 100Cr6 steel in combination with a counter body made of Teflon using a lubricated Stribeck test. In this test method, the COF is measured as a function of the Hersey number, a dimensionless number obtained from the sliding velocity multiplied with the dynamic viscosity and divided by the applied load. With increasing Hersey number, the lubricant film thickness between the sliding partners increases and three typical friction regimes can be identified: (I) boundary, (II) mixed lubricant and (III) hydrodynamic [2931] regime. Fig. 1 (a) illustrates a typical Stribeck curve. In the boundary regime the lubricant film thickness is smaller than 1.2 times the surface roughness. Due to interlocking surfaces of the sliding partners, a large COF occurs. With increasing Hersey number, e.g. by increasing sliding speed, the film thickness between base plate and counter body increases, thus the COF decreases (mixed regime). After reaching a COF minimum, a further increase of the Hersey number leads to an increased COF in the hydrodynamic regime. This can essentially be attributed to internal rheological effects of the lubricant. Any modification of the surface roughness and topography offers the possibility to influence this friction behavior in all three regimes [32].

\section{Experimental}

For laser surface processing we used a micro-machining station (MM200 USP, Optec) equipped with an ultrashort pulsed laser (Pharos 10-600-PP, Light Conversion) having a pulse duration of $220 \mathrm{fs}$ (FWHM) at a repetition rate of $300 \mathrm{kHz}$. For the generation of the micro and nanostructures, the fundamental emission wavelength of $1030 \mathrm{~nm}$ was employed. Fig 2 shows the experimental setup for the surface treatment. The energy of the laser was adjusted by an external attenuator based on a rotating wave plate and a polarizer. Using a half wave plate in front of the focusing unit, the linear polarization of the laser beam was rotated orthogonal to the onwards used scanning direction. A galvo scanner (RTA AR800, Newson) was used in combination with a telecentric lens ( $\mathrm{f}=100 \mathrm{~mm}$ ) to focus the beam onto the sample with a spot diameter of $36 \mu \mathrm{m}\left(1 / \mathrm{e}^{2}\right)$.

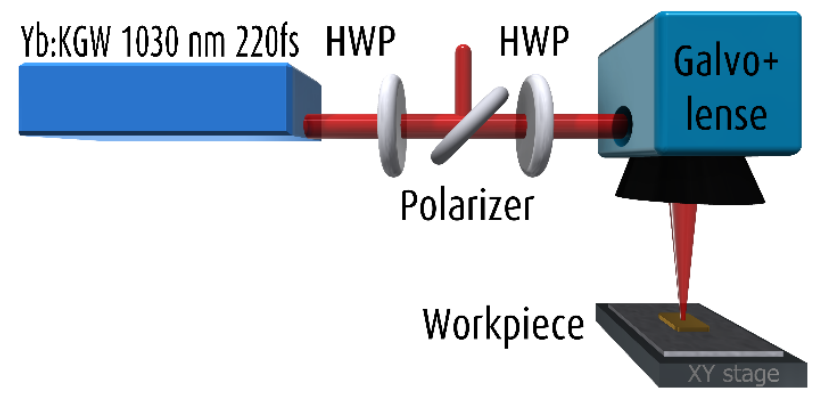

Fig. 2 Schematic laser system setup with Light Conversion Pharos 10-600. First, the half wave plate (HWP) and Polarizer allow energy adjustment; second, the HWP controls the orientation of the laser-induced periodic surface structures.

The geometrical dimensions of LSFL and the generated wear on the sliding partners were captured via laser scanning microscopy (VK-X200, Keyence) with subsequent image analysis via 2D Fourier transformation. Based on equation 1, the two-dimensional discrete Fourier transform of the microscope image can be calculated. 


$$
F(u, v)=\sum_{x=0}^{X-1} \sum_{y=0}^{Y-1} f(x, y) * e^{-i 2 \pi \frac{x u}{X}} * e^{-i 2 \pi \frac{y v}{Y}}
$$

Herein $\mathrm{u}$ and $\mathrm{v}$ are spatial frequencies in $\mathrm{x}$ and $\mathrm{y}$ directions, whereas $\mathrm{F}(\mathrm{u}, \mathrm{v})$ is the two-dimensional spectrum of the microscope image $f(x, y)$. $X$ and $Y$ represent the size of the image $f(x, y)$.

The disk for the Stribeck test is a round $100 \mathrm{Cr} 6$ steel disk with a diameter of $70 \mathrm{~mm}$ and a thickness of $6.6 \mathrm{~mm}$. Before the laser modification is carried out, the base plate was prepared by grinding and polishing with a multi directional polishing with several abrasive strength and afterwards polished with suspensions down to a grain size of $1 \mu \mathrm{m}$ to achieve a reference surface with a roughness $R_{a}$ of $14 \mathrm{~nm}$. To investigate the influence of laser induced periodic surface structures on the COF, concentric rings, filled with LIPSS were produced on the steel surface (c.f. Fig. $1 \mathrm{~b}$ and c).

The measurement of the COF was performed in a ballon-disk setup. We used the Universal Mechanical Tester from Bruker (UMT TriboLab) to perform the Stribeck test. The system is equipped with a rotation drive allowing rotation speeds up to $5000 \mathrm{rpm}$ and a second drive unit to apply a specific load of the ball on the base plate. The used force sensor allows measuring the load $\mathrm{F}_{\mathrm{L}}$ and the lateral force $\mathrm{F}_{\mathrm{X}}$ up to $5 \mathrm{~N}$. The resulting COF is calculated by $\mathrm{F}_{\mathrm{X}} / \mathrm{F}_{\mathrm{L}}$. We measured the friction behaviour of commercially available polytetrafluoroethylene (PTFE, Teflon) in a ball shape with a diameter of $6.3 \mathrm{~mm}$. The ball holder and the force sensor are fixed on a moveable stage along the radius of the disk. Thus either structured surfaces or reference surfaces in different tracks on concentric rings can be measured. During the complete Stribeck Test the rotation disk is covered with engine oil (5W40). A special liquid chamber allows a steady presence of lubricant between the disk and the ball

Due to the elastic deformation of the Teflon ball, different contact areas appear. Using a Hertzian deformation model of a sphere in contact with a flat sample, the diameter of the contact area is calculated to $151 \mu \mathrm{m}, 328 \mu \mathrm{m}$ and $562 \mu \mathrm{m}$ for $100 \mathrm{mN}, 1000 \mathrm{mN}$ and $5000 \mathrm{mN}$, respectively.

\section{Results and discussion}

\subsection{LSFL generation}
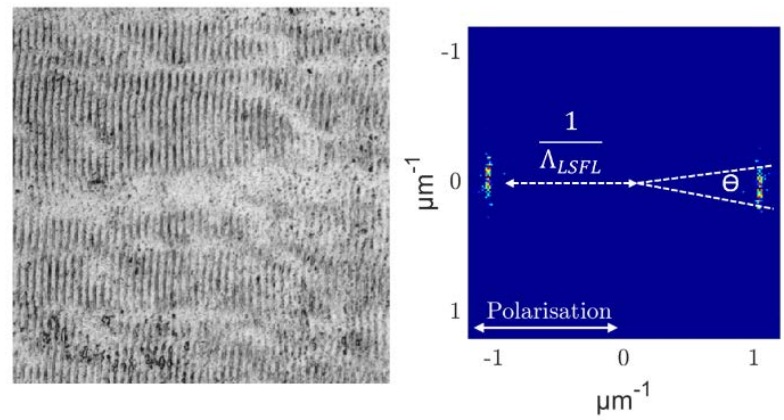

Fig. 3 Microscope image of LSFL (left) and corresponding transformed 2D FFT spectra (right) for the analysis of LSFL generated using $0.29 \mathrm{~J} / \mathrm{cm}^{2}$ and $80 \%$ overlap.

In the first step, the required laser parameters for continuous planar LSFL generation on $100 \mathrm{Cr} 6$ steel are determined with the laser fluence being varied between $0.15 \mathrm{~J} / \mathrm{cm}^{2}$ and $0.65 \mathrm{~J} / \mathrm{cm}^{2}$. The pulse overlap is investigated in a range of $70 \%$ to $90 \%$, resulting in a spatial pulse to pulse distance of $10.8 \mu \mathrm{m}$ to $3.6 \mu \mathrm{m}$ with the scanning speed between $3250 \mathrm{~mm} / \mathrm{s}$ and $1080 \mathrm{~mm} / \mathrm{s}$. Please note that for a homogenous energy deposition, the given pulse overlap holds for both, the consecutive laser pulses inside a scanning track and the overlap of the individual scanning tracks, i.e. the spatial distance between the deposited pulses equals the hatch pitch of the structured surface area.

To produce regular LSFL on the surface, the scanning direction is set orthogonal to the polarization of the laser light. Due to this method, the connection quality of LSFL between the individual scanning tracks is improved [7]. The influence of the laser fluence and the pulse to pulse overlap on the LSFL generation is evaluated by 2D FFT. A microscope image of LSFL covered surfaces and the corresponding 2D FFT spectra are shown in Fig. 3. Less pronounced LSFL with inferior homogeneity and quality are generated using a pulse overlap of $80 \%$ and a laser fluence of $0.29 \mathrm{~J} / \mathrm{cm}^{2}$. In the 2D FFT spectra this evidences in a recognizable opening angle $\theta$, which in turn can be considered as a quality measure for uniform LSFL. The distance between the center of the 2D FFT and the peak of the frequency sickle represents the mean of the spatial period of the generated LSFL.

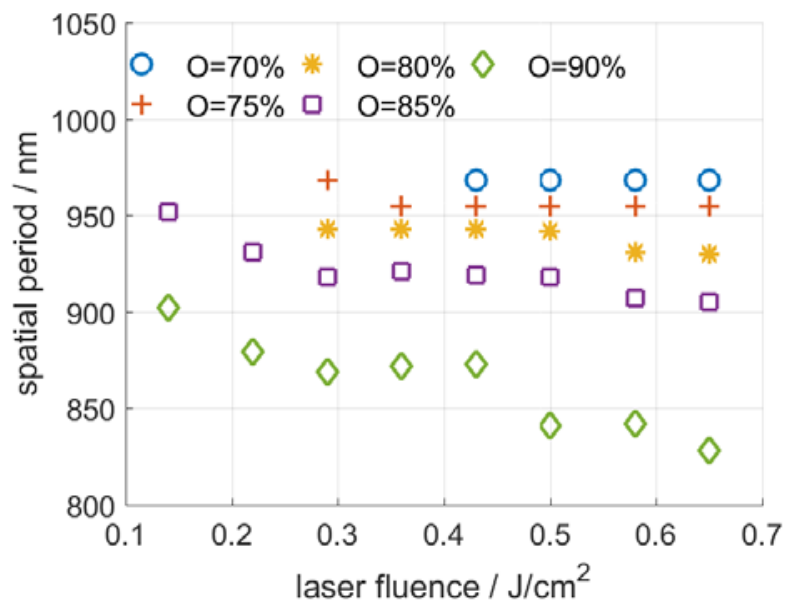

Fig. 4 Spatial period of LSFL for different pulse overlap values as a function of the laser fluence.

All investigated processing parameters lead to a complete LSFL coverage on the 100Cr6 steel surface. The general morphology is similar to the structures shown in Fig 3 and Fig 6. Rung et al. [14] determined a $|\sin (\mathrm{x})|$ - like surface profile description of LSFL on brass using an AFM measurement. Fig 4 shows the resulting spatial periods of LSFL on stainless steel. The inverse value of the 2D FFT centroid is plotted against the laser fluence for pulse overlap values of $70 \%, 75 \%, 80 \%, 85 \%$ and $90 \%$, respectively. With decreasing overlap, the required laser fluence increases to generate fully covered LSFL surfaces. With increasing laser fluence and increasing pulse overlap, the spatial period of LSFL decreases. This behavior can be explained by the generation of a surface plasma wave through the parametric decay of laser light [21] and a feedback mechanism based on a 
grating assisted SSP excitation and the incident laser radiation [22].

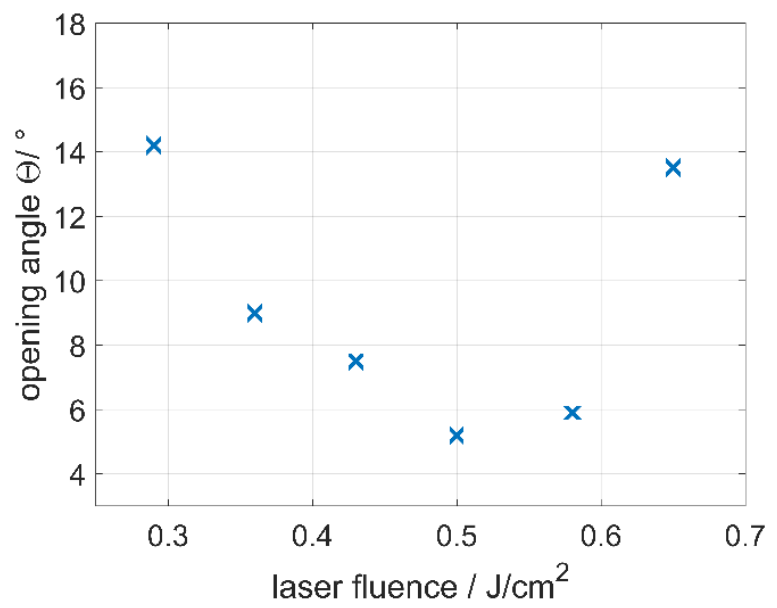

Fig. 5 Opening angle of FFT sickle as a function of the laser fluence for a pulse overlap of $80 \%$

The resulting opening angle of the 2D FFT sickle is shown in Fig. 5 for a pulse overlap of $80 \%$. A laser fluence of $0.29 \mathrm{~J} / \mathrm{cm}^{2}$ leads to an incomplete LSFL surface covering which results in a large spread angle. With increasing laser fluence the uniformity of the LSFL increases, i.e. $\theta$ decreases. The smallest spread angle can be achieved using a laser fluence of $0.5 \mathrm{~J} / \mathrm{cm}^{2}$. For a yet further increased fluence surface damage and burr formation is observed, deteriorating LSFL quality and uniformity.

\subsection{Tribological evaluation by Stribeck test}

As a result of section 3.1., continuous planar LSFL with high homogeneity are generated for the tribological tests using a laser fluence of $0.5 \mathrm{~J} \mathrm{~cm}^{2}$ and a pulse overlap of $80 \%$ (cf. Fig. 6). This parameter set results in LSFL having a period of 942nm (see Fig. 4). In comparison to this periodicity, the calculated contact area of the PTFE counter body (up to a diameter of $562 \mu \mathrm{m}$ for $5 \mathrm{~N}$ ) is much larger, excluding a direction depended friction behavior to be observable in our test. Three concentric rings with a width of $5 \mathrm{~mm}$ are filled with LSLF (c.f. orange rings in Fig. 1 b) with the unstructured, polished areas between these rings being used as the reference surface for the tribological test.

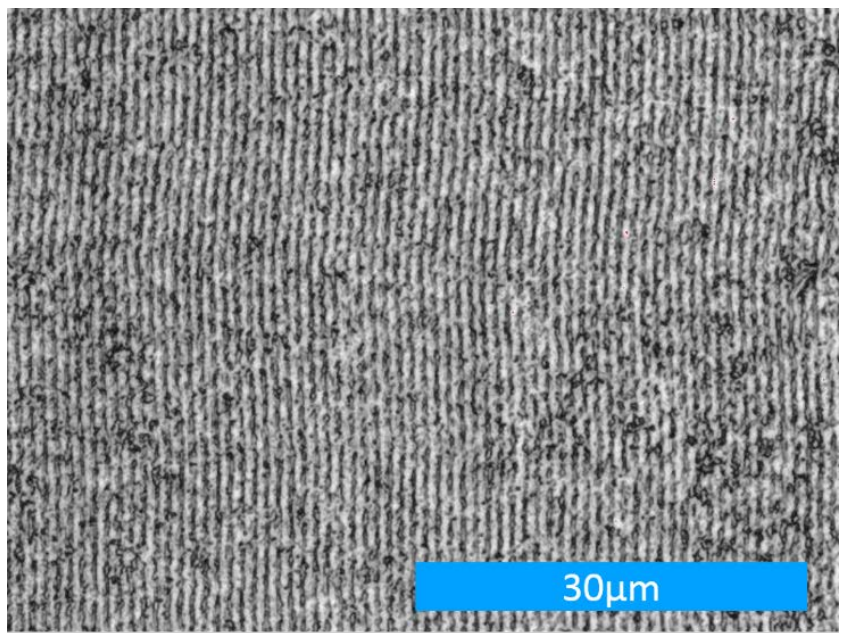

Fig. 6 Microscope image of LSFL on 100Cr6 steel using $0.5 \mathrm{~J} / \mathrm{cm}^{2}$ and $80 \%$ pulse overlap.
The Stribeck test is performed by subsequently increasing the rotation speed. The used steps are (1, 2, 5, 10, 20, 50, $100,500,1000,2000,3000,4000,5000) \mathrm{rpm}$. Each of this steps is performed for $60 \mathrm{~s}$ using a data acquisition rate of $100 \mathrm{~Hz}$, allowing collection of sufficient data for an accurate result. For each load force $(100 \mathrm{mN}, 1000 \mathrm{mN}$ and $5000 \mathrm{mN}$ ), a structured disc is prepared. By the application of three LSFL covered test tracks, the load specific Stribeck tests are performed three times with identical testing conditions for LSFL and reference surface, respectively. All following graphs are the result of the combination of this threefold measurement approach. The mean value and the standard deviation for each rotation speed step is calculated. By the combination of rotation speed and track radius the specific translation speed is calculated and plotted in the following Stribeck curves. For all evaluated normal loads, no wear on the disc surface, neither on the structured nor on the reference surface can be observed.

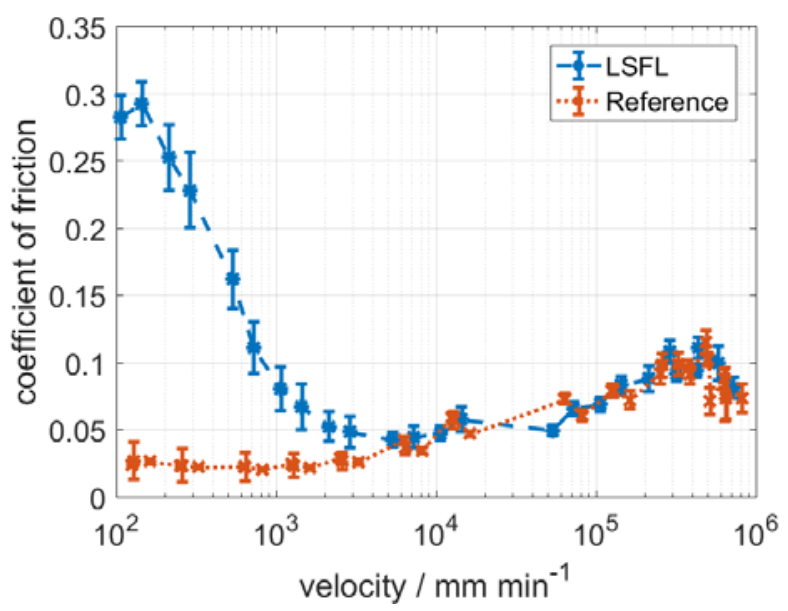

Fig. 7 Stribeck curve for LSFL covered surface (blue) and reference (orange) using a normal load of $100 \mathrm{mN}$.

Fig. 7 depicts the Stribeck curve for an applied normal load of $100 \mathrm{mN}$. The orange curve shows the coefficient of friction against the sliding velocity on the polished reference surface. Even for the slowest sliding velocity, the COF is very small, i.e. no distinctive stiction occurs, which is a typical behavior for Teflon on a smooth steel surface [33]. With increasing sliding speed, the COF increases to values of approx. 0.1 indicating the transition to full film hydrodynamic lubrication. The blue curve in Fig. 7 represents the friction behavior of the LSFL covered surface. Contrary to the reference surface, a pronounced stiction is caused by the laser nanostructures, i.e. increased boundary layer lubrication. The COF is increased by a factor of approx. 10 for the slowest sliding speed around $10^{2} \mathrm{~mm} / \mathrm{min}$. With increasing velocity, the COF decreases and reaches a local minimum at 0.04 for $5300 \mathrm{~mm} / \mathrm{min}$, almost coincident with the COF of the reference. As outlined in the introduction, this general characteristic of lubricant friction can be attributed to the increasing lubricant film thickness between the two sliding partners. With further increasing velocity, the Stribeck curve of the LSFL structured surface is almost identical to the one of the reference surface. Apparently, for a normal load of $100 \mathrm{mN}$, the nanostructures do not influence the COF in this 
hydrodynamic regime by a dominating full film lubrication. Thus, the lubricant film thickness appears to be sufficiently larger than the modulation depth of the LSFL and the lubricated contact does not suffer from a temporal short lubricant supply, i.e. no starvation effects hindering the lubricant to fill the contact inlets between the substrate and the tribometer ball adequately [34].

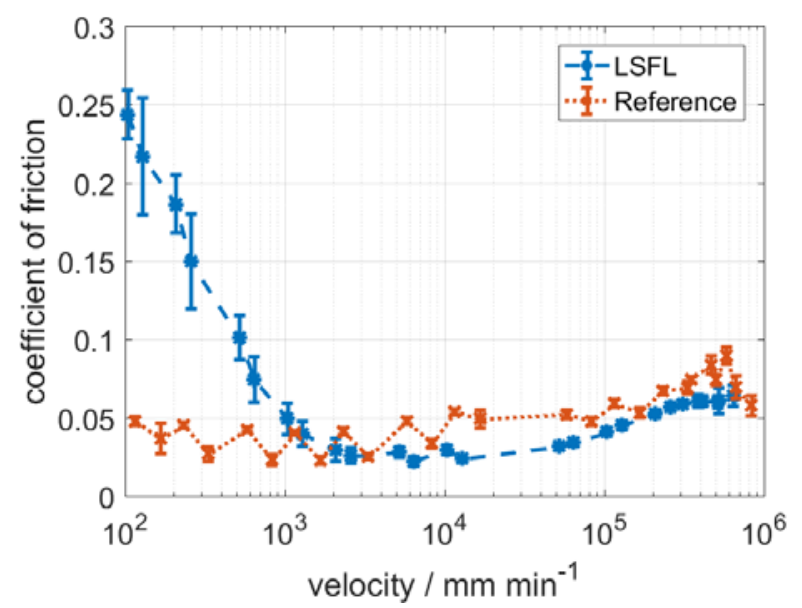

Fig. 8 Stribeck curve for LSFL covered surface (blue) and reference (orange) using a normal load of $1000 \mathrm{mN}$.

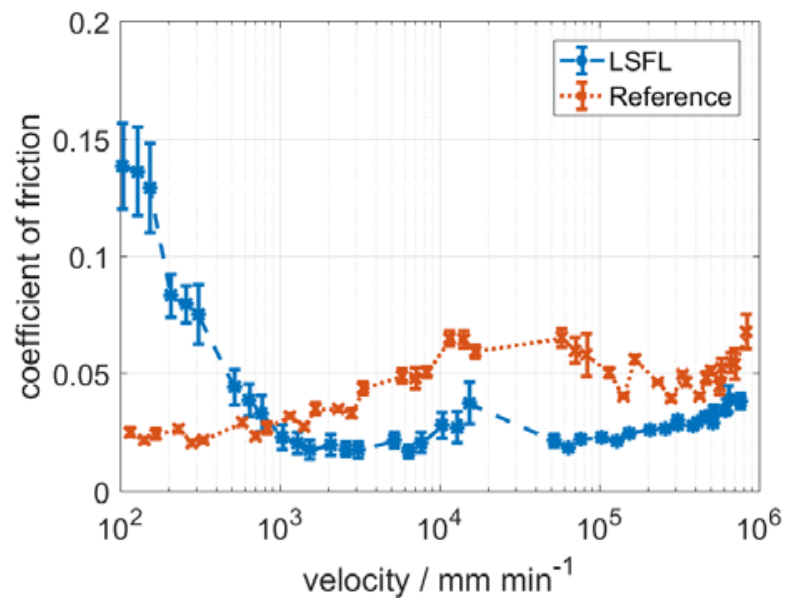

Fig. 9 Stribeck curve for LSFL covered surface (blue) and reference (orange) using a normal load of $5000 \mathrm{mN}$.

The Stribeck curves for an increased normal load of $1000 \mathrm{mN}$ are shown in Fig. 8, revealing an almost similar behavior of a stronger stiction of LSFL covered surfaces for low velocities (by a factor of 5) and decreasing COF with increasing velocity, accomplishing the COF of the unstructured, polished reference surface in the range between 1000 to about $3000 \mathrm{~mm} / \mathrm{min}$. For higher velocities, however, friction on the reference surface exceeds the COF of the LSFL covered specimen for sliding speeds above $10000 \mathrm{~mm} / \mathrm{min}$. This indicates that under this higher load full film lubrication is not yet fully formed for the LSFL structured surfaces for these sliding speeds as the generated nanostructures may impede undisturbed engine oil film lubrication.

With a further increase of the normal load to $5000 \mathrm{mN}$, we find an even more pronounced effect of the LSFL structuring on the COF, as shown in Fig. 9. Again, for lower velocities the COF is increased by a factor of 5 for the laser nanostructured surface as compared to the reference polished surface. However, with increasing velocity the COF for the LSFL structured specimen equals the one of the reference surface, again by trend as compared to the results shown in Fig. 7 and Fig. 8, for lower speeds already in the range of below $1000 \mathrm{~mm} / \mathrm{s}$, with the COF continuously being lower for the LSFL structured surfaces. Again, this indicates changes in the governing mixed lubricant and hydrodynamic regime, namely a shift of the transition from the mixed to the hydrodynamic regime to higher velocities with increasing load for LSFL structured surfaces.

\section{Conclusion}

We report on tribological surface functionalization upon laser induced periodic surface structures on 100Cr6 steel. In combination with PTFE, a ball on disk setup is used to perform a Stribeck test in an engine oil environment. LSFL covered surfaces lead to a PTFE untypical stiction increase. For a test load of $100 \mathrm{mN}$, a 10 -fold increase of the stiction is caused by LSFL. From a application point of view, this effect is beneficial for all kinds of force and torque transmission. With increasing sliding speed, the coefficient of friction decreases. The slope of this curve increases with increasing test load. Using $5000 \mathrm{mN}$ load, it is possible to reduce lubricated sliding friction for velocities between $10^{3} \mathrm{~mm} / \mathrm{min}$ and $10^{6} \mathrm{~mm} / \mathrm{min}$. In this regime, the LSFL covered surfaces could lead to a decreased energy loss for moving parts and also a decreased wear generation.

\section{Acknowledgment}

This work has been supported within the BMBF project LAMILE (grant 03FH030IX4).

\section{References}

[1] K. Holmberg and A. Erdemir: Friction, 5, (2017), 263.

[2] A. Ancona, G. Carbone, M. de Filippis, A. Volpe and

P. M. Lugarà: Adv. Opt. Technol., 3, (2014), 42.

[3] F. P. Mezzapesa, M. Scaraggi, G. Carbone, D. Sorgente, A. Ancona and P. M. Lugarà: Physics Procedia, 41, (2013), 677.

[4] M. Scaraggi, F. P. Mezzapesa, G. Carbone, A. Ancona, D. Sorgente and P. M. Lugarà: Tribol. Int., 75, (2014), 123.

[5] A. Ancona, G. Joshi, A. Volpe, M. Scaraggi, P. Lugarà and G. Carbone: Lubricants, 5, (2017), 41.

[6] B. K. Nayak and M. C. Gupta: Opt. Lasers Eng., 48, (2010), 940.

[7] I. Gnilitskyi, T. J.-Y. Derrien, Y. Levy, N. M. Bulgakova, T. Mocek and L. Orazi: Sci. Rep., 7, (2017), 8485. [8] S. Schwarz, S. Rung and R. Hellmann: Appl. Phys. Lett., 108, (2016), 181607.

[9] J. Bonse, J. Krüger, S. Höhm and A. Rosenfeld: J. La-

(Received: June 24, 2018, Accepted: October 7, 2018)

ser Appl., 24, (2012), 42006.

[10] T. J.-Y. Derrien, J. Krüger, T. E. Itina, S. Höhm, A. Rosenfeld and J. Bonse: Appl. Phys. A, 117, (2014), 77.

[11] M. Birnbaum: J. Appl. Phys., 36, (1965), 3688.

[12] A. Y. Vorobyev and C. Guo: Laser Photonics Rev., 7, (2013), 385.

[13] G. Li, J. Li, Y. Hu, C. Zhang, X. Li, J. Chu and W. Huang: Appl. Phys. A, 118, (2015), 1189. 
[14] S. Rung, S. Schwarz, B. Götzendorfer, C. Esen and R. Hellmann: Appl. Sci., 8, (2018), 700.

[15] D. van Ta, A. Dunn, T. J. Wasley, J. Li, R. W. Kay, J. Stringer, P. J. Smith, E. Esenturk, C. Connaughton and J. D. Shephard: Appl. Surf. Sci., 371, (2016), 583.

[16] O. Raimbault, S. Benayoun, K. Anselme, C. Mauclair, T. Bourgade, A.-M. Kietzig, P.-L. Girard-Lauriault, S. Valette and C. Donnet: Mater. Sci. Eng. C Mater. Biol. Appl., 69, (2016), 311.

[17] K. Wallat, D. Dörr, R. Le Harzic, F. Stracke, D. Sauer, M. Neumeier, A. Kovtun, H. Zimmermann and M. Epple:

J. Laser Appl., 24, (2012), 42016.

[18] C. Gachot, A. Rosenkranz, L. Reinert, E. RamosMoore, N. Souza, M. H. Müser and F. Mücklich: Tribol Lett, 49, (2013), 193.

[19] A. Ancona, G. Carbone, M. Scaraggi, F. P. Mezzapesa, D. Sorgente and P. M. Lugarà: SPIE LASE. San Francisco, California, United States, SPIE Proceedings (2014, 896806. [20] E. V. Golosov, V. I. Emel'yanov, A. A. Ionin, Y. R. Kolobov, S. I. Kudryashov, A. E. Ligachev, Y. N. Novoselov, L. V. Seleznev and D. V. Sinitsyn: Jetp Lett., 90, (2009), 107.

[21] K. Okamuro, M. Hashida, Y. Miyasaka, Y. Ikuta, S. Tokita and S. Sakabe: Phys. Rev. B, 82, (2010), 1.

[22] J. Bonse and J. Krüger: J. Appl. Phys., 108, (2010), 34903.

[23] F. Preusch, S. Rung and R. Hellmann: J. Laser. Micro/Nanoengin., 11, (2016), 137.

[24] R. Stribeck: Z. Verein. Deut. Ing, 46, (1902), 38. [25] J. Bonse, R. Koter, M. Hartelt, D. Spaltmann, S. Pentzien, S. Höhm, A. Rosenfeld and J. Krüger: Appl. Phys. A, 117, (2014), 103.

[26] J. Eichstädt, G. Römer and A. H. i. Veld: Physics Procedia, 12, (2011), 7.

[27] J. Bonse, S. V. Kirner, M. Griepentrog, D. Spaltmann and J. Krüger: Materials (Basel), 11, (2018).

[28] Z. Wang, Q. Zhao and C. Wang: Micromachines, 6, (2015), 1606.

[29] D. Braun, C. Greiner, J. Schneider and P. Gumbsch: Tribol. Int., 77, (2014), 142.

[30] A. Kovalchenko, O. Ajayi, A. Erdemir, G. Fenske and I. Etsion: Tribol. Int., 38, (2005), 219.

[31] J. W. Robinson, Y. Zhou, P. Bhattacharya, R. Erck, J. Qu, J. T. Bays and L. Cosimbescu: Sci. Rep., 6, (2016), 18624.

[32] A. Martini, D. Zhu and Q. Wang: Tribol Lett, 28, (2007), 139.

[33] P. M. Dickens, J. L. Sullivan and J. K. Lancaster: Wear, 112, (1986), 273.

[34] R. Kumar, P. Kumar and M. Gupta: International Journal of Advancements in Technology, 1, (2010), 73.

(Received: June 24, 2018, Accepted: October 7, 2018) 